\title{
DIRECT THEOREMS OF TRIGONOMETRIC APPROXIMATION FOR VARIABLE EXPONENT LEBESGUE SPACES
}

\author{
RAMAZAN AKGÜN
}

ABSTRACT. Jackson type direct theorems are considered in variable exponent Lebesgue spaces $L^{p(x)}$ with exponent $p(x)$ satisfying $1 \leq \operatorname{essinf}_{x \in[0,2 \pi]} p(x)$, $\operatorname{ess}_{\sup _{x \in[0,2 \pi]}} p(x)<\infty$, and the Dini-Lipschitz condition. Jackson type direct inequalities of trigonometric approximation are obtained for the modulus of smoothness based on one sided Steklov averages

$$
\mathfrak{Z}_{v} f(\cdot):=\frac{1}{v} \int_{0}^{v} f(\cdot+t) d t
$$

in these spaces. We give the main properties of the modulus of smoothness

$$
\Omega_{r}(f, v)_{p(\cdot)}:=\left\|\left(\mathbf{I}-\mathfrak{Z}_{v}\right)^{r} f\right\|_{p(\cdot)} \quad(r \in \mathbb{N})
$$

in $L^{p(x)}$, where $\mathbf{I}$ is the identity operator. An equivalence of the modulus of smoothness and Peetre's $K$-functional is established.

\section{INTRODUCTION}

The main purpose of this work is to obtain a Jackson type direct theorem for functions in generalized Lebesgue spaces $L^{p(\cdot)}$ with variable exponent $p(x)$ : $[0,2 \pi) \rightarrow[1, \infty)$, satisfying the Dini-Lipschitz condition and

$$
1 \leq \mathbf{p}:=\operatorname{essinf}_{x \in[0,2 \pi)} p(x) \leq \operatorname{ess}_{\sup _{x \in[0,2 \pi)}} p(x)=: \mathbb{P}<\infty .
$$

The main difficulty related to $L^{p(\cdot)}$ theory is that the spaces $L^{p(\cdot)}$ are, in general, not translation invariant, see e.g. [10, Proposition 3.6.1]. This inadequacy and the structure of $L^{p(\cdot)}$ cause some additional problems. For example Young's Convolution inequality and Cavalieri's equality do not hold in the spaces $L^{p(\cdot)}$. Maximal, Poincáre, and Sobolev inequalities do not hold in a modular form in $L^{p(\cdot)}$ either. Interpolation is not so useful in $L^{p(\cdot)}$. Solutions of the $p(\cdot)$-Laplace equation are not scalable.

To obtain a Jackson type inequality in $L^{p(\cdot)}$ we can not use the classical modulus of smoothness $\omega_{r}(f, \cdot)_{p}$ because the classical translation $\mathbf{t}_{a} f(x):=f(x+a)$ of

2010 Mathematics Subject Classification. Primary 42A10; Secondary 41A17.

Key words and phrases. Lebesgue spaces with variable exponent; $K$-functional; Steklov mean; Best approximation.

This work was supported by Balikesir University Research Project "A modulus of smoothness in $L^{p(x) "}$ in 2019 . 
$f(\cdot)$, where $a \in \mathbb{R}$, may not be in the class $L^{p(\cdot)}$ even if $f$ is in $L^{p(\cdot)}$, see e.g. [10, Proposition 3.6.1]. So the classical modulus of smoothness $\omega_{r}$ may not be suitable for functions $f \in L^{p(\cdot)}$. Instead of using the classical translation operator $f \mapsto \mathbf{t}_{a} f$, $a \in \mathbb{R}$, we will consider the one sided Steklov average

$$
\mathfrak{Z}_{v} f(x):=\frac{1}{v} \int_{0}^{v} \mathbf{t}_{u} f(x) d u, \quad v>0 ; \quad \mathfrak{Z}_{0} f:=f
$$

in $L^{p(\cdot)}[22,23,2$, 24]. Under some condition on $p(x)$ Sharapudinov [22, 23] obtained that the family of operators $\left\{\mathfrak{Z}_{v} f\right\}_{0<v<1}$ is uniformly bounded on $L^{p(\cdot)}$ (see Theorem 1.1 below).

Let $\mathbf{T}:=[0,2 \pi)$ and let $\mathcal{E}$ be the class of Lebesgue measurable functions $p(x)$ : $\mathbf{T} \rightarrow[1, \infty)$ such that $1 \leq \mathbf{p} \leq \mathbb{P}<\infty$. The variable exponent $p(x)$ is said to satisfy the Dini-Lipschitz property on $\mathbf{T}$ ([22]) if there exists a (Dini-Lipschitz) constant $\mathbb{A}>0$, depending only on $p(x)$, such that

$$
|p(x)-p(y)| \ln 2 \pi|x-y|^{-1} \leq \mathbb{A}<\infty,
$$

for all $x, y \in \mathbf{T}$ with $x \neq y$. We will denote by $\mathcal{P}$ the class of those exponents $p \in \mathcal{E}$ that satisfy the Dini-Lipschitz property 1.1 on $\mathbf{T}$. We define the variable exponent Lebesgue space $L^{p(\cdot)}$ as the collection of $2 \pi$-periodic Lebesgue measurable functions $f: \mathbf{T} \rightarrow \mathbb{R}$ having the norm

$$
\|f\|_{p(\cdot)}:=\inf \left\{\alpha>0: \int_{\mathbf{T}}\left|\frac{f(x)}{\alpha}\right|^{p(x)} d x \leq 1\right\}<\infty,
$$

where $p \in \mathcal{E}$. The space $L^{p(\cdot)}$ is a Banach space. If $p \in \mathcal{P}, p^{\prime}(x):=p(x) /(p(x)-1)$ for $p(x)>1$, and $p^{\prime}(x):=\infty$ for $p(x)=1$, then Hölder's inequality

$$
\int_{\mathbf{T}}|f(x) g(x)| d x \leq\left(1+\frac{1}{\mathbf{p}}-\frac{1}{\mathbb{P}}\right)\|f\|_{p(\cdot)}\|g\|_{p^{\prime}(\cdot)}=\mathbb{H}\|f\|_{p(\cdot)}\|g\|_{p^{\prime}(\cdot)}
$$

holds when $f \in L^{p(\cdot)}$ and $g \in L^{p^{\prime}(\cdot)}$. We know that

$$
\|f\|_{p(\cdot)} \leq(2 \pi+1)\|f\|_{q(\cdot)}=\mathbb{D}\|f\|_{q(\cdot)}
$$

for $p \in \mathcal{E}$ and $1 \leq p(x) \leq q(x) \leq \bar{q}:=\operatorname{ess}_{\sup _{x \in \mathbf{T}}} q(x)<\infty$ a.e. on $\mathbf{T}$.

Theorem 1.1. If $p \in \mathcal{P}, f \in L^{p(\cdot)}$, and $0<v \leq 1$, then

$$
\left\|\mathfrak{Z}_{v} f\right\|_{p(\cdot)} \leq\left\|\mathfrak{Z}_{v}\right\|_{L^{p(\cdot)} \rightarrow L^{p(\cdot)}}\|f\|_{p(\cdot)},
$$

where $\|f\|_{p(\cdot)}$ is the Luxemburg norm 1.2 of $f$ and

$$
\left\|\mathfrak{Z}_{v}\right\|_{L^{p(\cdot)} \rightarrow L^{p(\cdot)}} \leq \mathbb{B}:=\left(\frac{136}{25}\right)^{\mathbb{A}}(2 \pi+1)^{\frac{\mathbb{A}}{\ln 2}+\mathbb{P}} .
$$

Inequality (1.4) was obtained in [22, 23]; 1.5 can be proved similarly.

After this result one can define modulus of smoothness. For $p \in \mathcal{P}, f \in L^{p(\cdot)}$, and $0<v \leq 1$, we set

$$
\Omega_{r}(f, v)_{p(\cdot)}:=\left\|\left(\mathbf{I}-\mathfrak{Z}_{v}\right)^{r} f\right\|_{p(\cdot)}, \quad r \in \mathbb{N} .
$$


For $r=1$ see 24. Note that modulus of smoothness is the main notion in the approximation theory and it is used in many of the approximation results such as Jackson type direct theorems, Salem-Stechkin type inverse theorems, Marchaud type inequalities, etc. First of all the set of trigonometric polynomials is a dense subset $([23$, Theorems 6.1 and 6.2$])$ of $L^{p(\cdot)}$ when $p \in \mathcal{P}$. This allows us to consider approximation problems in $L^{p(\cdot)}$. Jackson type inequalities in $L^{p(\cdot)}$ were investigated by several mathematicians. For example in 14] Israfilov and Testici obtained the following estimate:

$$
\begin{aligned}
& \text { If } f \in L^{p(\cdot)}, p \in \mathcal{P} \text {, then } \\
& E_{n}(f)_{p(\cdot)}:=\inf _{t \in \Pi_{n}}\|f-t\|_{p(\cdot)}=\mathcal{O}(1) \sup _{0 \leq h \leq 1 / n}\left\|\frac{1}{h} \int_{0}^{h}\left(\mathbf{I}-\mathbf{t}_{u}\right)^{r} f(\cdot) d u\right\|_{p(\cdot)}
\end{aligned}
$$

holds for $n \in \mathbb{N}$, with constant depending only on $p$, where $\Pi_{n}$ is the class of trigonometric polynomials of degree not greater than $n$. that

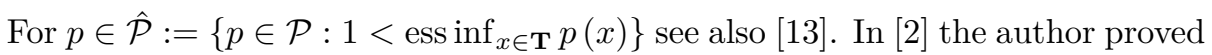

$$
\text { If } f \in L^{p(\cdot)}, r>0, p \in \hat{\mathcal{P}} \text {, then }
$$

$$
E_{n}(f)_{p(\cdot)}=\mathcal{O}(1) \sup _{0 \leq h_{i}, t \leq 1 / n}\left\|\left(\mathbf{I}-\Phi_{h_{1}}\right) \cdots\left(\mathbf{I}-\Phi_{h_{\lfloor r\rfloor}}\right)\left(\mathbf{I}-\Phi_{t}\right)^{\{r\}} f\right\|_{p(\cdot)}
$$

holds for $n \in \mathbb{N}$, with constant depending only on $p, r$, where

$$
\Phi_{h} f(x):=\frac{1}{h} \int_{x-h / 2}^{x+h / 2} f(t) d t,
$$

$\lfloor r\rfloor:=\max \{n \in \mathbb{N}: n \leq x\},\{r\}:=r-\lfloor r\rfloor, \mathbf{I}$ is the identity operator, and $\left(\mathbf{I}-\Phi_{t}\right)^{\{r\}}$ is the fractional binomial series expansion of $\mathbf{I}-\Phi_{t}$.

Here, and in what follows, $A=\mathcal{O}(1) B$ means that $A / B$ is less than or equal to some constant, depending on essential parameters only.

In [24, 28] Sharapudinov and Volosivets considered the following type of modulus of smoothness of order $r \in \mathbb{N}$ :

$$
\sup _{0 \leq v_{i} \leq \delta}\left\|\left(\mathbf{I}-\mathfrak{Z}_{v_{1}}\right) \cdots\left(\mathbf{I}-\mathfrak{Z}_{v_{r}}\right) f\right\|_{p(\cdot)}
$$

in the right hand side of $(1.7)$ :

$$
\begin{aligned}
& \text { If } f \in L^{p(\cdot)}, p \in \mathcal{P} \text {, then }([24, r=1],[28, r \geq 1]) \\
& \qquad E_{n}(f)_{p(\cdot)}=\mathcal{O}(1) \sup _{0 \leq v_{i} \leq \delta}\left\|\left(\mathbf{I}-\mathfrak{Z}_{v_{1}}\right) \cdots\left(\mathbf{I}-\mathfrak{Z}_{v_{r}}\right) f\right\|_{p(\cdot)}
\end{aligned}
$$

holds for $n, r \in \mathbb{N}$, with constant depending only on $p, r$.

In the present work we will consider a more natural and smaller modulus of smoothness:

$$
\Omega_{r}(f, v)_{p(\cdot)}:=\left\|\left(\mathbf{I}-\mathfrak{Z}_{v}\right)^{r} f\right\|_{p(\cdot)}, \quad v \geq 0
$$


for $f \in L^{p(\cdot)}$ and $p \in \mathcal{P}$. Our result, given below, refines the Jackson type estimates (1.7) and 1.8.

Theorem 1.2. We suppose that $p \in \mathcal{P}, f \in L^{p(\cdot)}$, and $r \in \mathbb{N}$. In this case we have inequalities

$$
\begin{gathered}
E_{n}(f)_{p(\cdot)} \leq \mathbb{C} \Omega_{r}\left(f, \frac{1}{n}\right)_{p(\cdot)}, \\
\left(\prod_{s=1}^{n} E_{s}(f)_{p(\cdot)}\right)^{1 / n} \leq \frac{\mathbb{C}^{2}(2 e[1+\mathbb{B}])^{r}}{\mathbb{B}} \Omega_{r}\left(f, \frac{1}{n}\right)_{p(\cdot)},
\end{gathered}
$$

where $\mathbb{C}=2 \mathbb{B}\left[\max \left\{216 \mathbb{B} \mathbb{H} \sum_{j=0}^{r-1} \mathbb{B}^{j}, 2[1+108 \mathbb{B H}+432 \mathbb{B H} \ln 2]\right\}\right]^{r}$.

For the constant exponent case $p(x)=p, 1 \leq p \leq \infty$, the $L^{p}$ modulus of smoothness (1.6) was considered by Trigub in 1968 [27] for $r=1,1 \leq p \leq \infty$, and by Ditzian and Ivanov [11] for $r \in \mathbb{N}, 1 \leq p \leq \infty$. In the case $r=1, p \in \mathcal{P}$, a Jackson type inequality was obtained in [24] by Sharapudinov (see (1.8) and [28]). For the constant exponent case $p(x)=p$, in $L^{p}$ the inequality 1.10 was obtained by Natanson and Timan (see [21]) for $r \in \mathbb{N}, p=\infty$.

Let $X$ be a Banach space with norm $\|\cdot\|_{X}$. By $X^{r}$ we denote the class of functions $f \in X$ such that $f^{(r-1)}$ is absolutely continuous and $f^{(r)} \in X$. When $r \in \mathbb{N}, p \in \mathcal{P}$, and $X=L^{p(\cdot)}$ we will denote $W_{p(\cdot)}^{r}:=X^{r}$.

The following second type Jackson inequality holds:

Theorem 1.3. Suppose that $r, k, n \in \mathbb{N}, p \in \mathcal{P}$, and $f \in W_{p(\cdot)}^{r}$. Then the following inequality holds:

$$
n^{r} E_{n}(f)_{p(\cdot)} \leq \mathbb{C}\left(\mathbb{B}\left(1+\mathbb{D}(2 \pi)^{\frac{1}{p}-1}\right)\right)^{r} \Omega_{k}\left(f^{(r)}, \frac{1}{n}\right)_{p(\cdot)} .
$$

One of the results of this paper is the following theorem, which contains an equivalence of $\Omega_{r}$ and Peetre's $K$-functional.

Theorem 1.4. If $r \in \mathbb{N}, p \in \mathcal{P}, f \in L^{p(\cdot)}$, then the equivalence

$$
\left\|\left(\mathbf{I}-\mathfrak{Z}_{v}\right)^{r} f\right\|_{p(\cdot)} \approx \inf \left\{\|f-g\|_{p(\cdot)}+v^{r}\left\|g^{(r)}\right\|_{p(\cdot)}: g \in W_{p(\cdot)}^{r}\right\}
$$

holds for $v>0$.

If $A(t)=\mathcal{O}(B(t))$ and $B(t)=\mathcal{O}(A(t))$, we will write $A(t) \approx B(t)$.

Corollary 1.5. If $k \in \mathbb{N}, p \in \mathcal{P}, f \in L^{p(\cdot)}$, then

$$
\Omega_{k}(f, \lambda v)_{p(\cdot)}=\mathcal{O}(1)(1+\lfloor\lambda\rfloor)^{k} \Omega_{k}(f, v)_{p(\cdot)}, \quad v, \lambda>0,
$$

and

$$
\frac{\Omega_{k}(f, v)_{p(\cdot)}}{v^{k}}=\mathcal{O}(1) \frac{\Omega_{k}(f, \delta)_{p(\cdot)}}{\delta^{k}}, \quad 0<\delta \leq v,
$$

where $\lfloor z\rfloor:=\max \{y \in \mathbb{Z}: y \leq z\}$, with constants depending only on $k, p$. 
The rest of the present work is organized as follows. In Section 2 we give upper estimates for the operator norm of Steklov and Jackson operators. Section 3 contains the main properties of the modulus of smoothness $\Omega_{r}$ and some integral operators. In Section 4 we give a transference result. In Section 5 we give the proof of the equivalence of the modulus of smoothness $\Omega_{r}$ and Peetre's $K$-functional $K_{r}$. Finally, Section 6 contains the proof of the refined Jackson inequality and other proofs.

In what follows, letters $\mathbf{c}, \mathbf{p}, \mathbb{A}, \mathbb{B}, \mathbb{C}, \ldots$ will stand for certain positive constants and these will not change in different places.

\section{UPPER ESTimates FOR the OPERATOR NORM OF Steklov AND JACKSON OPERATORS}

Theorem 2.1 ([23]). If $p \in \mathcal{P}, \vartheta>0,1 \leq \lambda<\infty,|\tau| \leq \pi \lambda^{-\vartheta}$, then the family of operators $\left\{\mathcal{S}_{\lambda, \tau}\right\}_{1 \leq \lambda<\infty}$, defined by

$$
\mathcal{S}_{\lambda, \tau} f(x)=\lambda \int_{x+\tau-1 /(2 \lambda)}^{x+\tau+1 /(2 \lambda)} f(u) d u
$$

is uniformly bounded (in $\lambda$ and $\tau$ ) in $L^{p(\cdot)}$ :

$$
\left\|\mathcal{S}_{\lambda, \tau} f\right\|_{p(\cdot)} \leq\left\|\mathcal{S}_{\lambda, \tau}\right\|_{L^{p(\cdot)} \rightarrow L^{p(\cdot)}}\|f\|_{p(\cdot)}
$$

with

$$
\left\|\mathcal{S}_{\lambda, \tau}\right\|_{L^{p(\cdot)} \rightarrow L^{p(\cdot)}} \leq \mathbb{B} .
$$

We point out that 2.1] was obtained in 223 and 2.2 can be obtained in the same way.

Let $p \in \mathcal{P}, f \in L^{p(\cdot)}, 0<v \leq 1$. We note that

$$
\mathfrak{Z}_{v} f(\cdot)=\frac{1}{v} \int_{0}^{v} \mathbf{t}_{u} f(\cdot) d u=\mathcal{S}_{\frac{1}{v}, \frac{v}{2}} f(\cdot) .
$$

Let $n \in \mathbb{N}$ and

$$
D_{n} f(x):=\frac{1}{\pi} \int_{\mathbf{T}} \mathbf{t}_{-u} f(x) \mathcal{J}_{2,\left\lfloor\frac{n}{2}\right\rfloor+1}(u) d u \in \Pi_{n}
$$

be the Jackson operator (polynomial) where $\mathcal{J}_{2, n}$ is the Jackson kernel

$$
\mathcal{J}_{2, n}(x):=\frac{1}{\varkappa_{2, n}}\left(\frac{\sin (n x / 2)}{\sin (x / 2)}\right)^{4}, \quad \varkappa_{2, n}:=\frac{1}{\pi} \int_{-\pi}^{\pi}\left(\frac{\sin (n t / 2)}{\sin (t / 2)}\right)^{4} d t .
$$

It is known ([12, p. 147]) that

$$
\frac{3}{2 \sqrt{2}} n^{3} \leq \varkappa_{2, n} \leq \frac{5}{2 \sqrt{2}} n^{3}
$$


The Jackson kernel $\mathcal{J}_{2, n}$ satisfies the relations

$$
\begin{gathered}
\frac{1}{\pi} \int_{\mathbf{T}} \mathcal{J}_{2, n}(u) d u=1 \\
\left|\mathcal{J}_{2, n}(u)\right| \leq \frac{2 \sqrt{2}}{3} \pi^{4}, \quad n^{-3 / 4} \leq u \leq \pi \\
\max _{u \in \mathbf{T}}\left|\mathcal{J}_{2, n}(u)\right| \leq\left(\pi 2^{-1}\right)^{4} n \\
\frac{1}{\pi} \int_{0}^{\pi} u \mathcal{J}_{2, n}(u) d u \leq \frac{1}{2 n}
\end{gathered}
$$

The next theorem was obtained in [24].

Theorem 2.2. If $p \in \mathcal{P}$ and $f \in L^{p(\cdot)}$, then the sequence of Jackson operators $\left\{D_{n} f\right\}_{1 \leq n<\infty}$ is uniformly bounded (in $n$ ) in $L^{p(\cdot)}$ :

$$
\left\|D_{n} f\right\|_{L^{p(\cdot)}} \leq\left\|D_{n}\right\|_{L^{p(\cdot)} \rightarrow L^{p(\cdot)}}\|f\|_{L^{p(\cdot)}},
$$

where

$$
\begin{aligned}
& \left\|D_{n}\right\|_{L^{p(\cdot)} \rightarrow L^{p(\cdot)}} \\
& \leq \mathbb{E}:=\left[\frac{\pi^{4}}{32}\right]^{\frac{4 \mathbb{A}}{\ln 2^{3 \mathbb{P}}}}\left[\frac{68}{25}\right]^{\frac{4 \mathbb{A}}{3 \mathbb{P}}}\left[\frac{45 \pi^{5} \mathbb{D}}{16}+\mathbb{H}\left[\frac{15 \pi^{5} \mathbb{D}}{16}\right]^{\mathbb{P}} 3\right]^{\frac{1}{\mathbb{P}}}+\left[\frac{2 \sqrt{2} \mathbb{D}}{3 \pi^{-4}}\right](2 \pi)^{\frac{1}{\mathbb{P}}} .
\end{aligned}
$$

\section{Modulus of Smoothness}

By Theorem 1.1 we have

$$
\left\|\left(\mathbf{I}-\mathfrak{Z}_{v}\right)^{k} f\right\|_{p(\cdot)} \leq(1+\mathbb{B})^{k}\|f\|_{p(\cdot)} .
$$

For $k \in \mathbb{N}$ we define the modulus of smoothness of $f \in L^{p(\cdot)}, p \in \mathcal{P}$, as

$$
\Omega_{k}(f, v)_{p(\cdot)}:=\left\|\left(\mathbf{I}-\mathfrak{Z}_{v}\right)^{k} f\right\|_{p(\cdot)}, \quad v>0 .
$$

From [28, (3.2) and Corollary 2] we have

Lemma 3.1. Let $p \in \mathcal{P}, k \in \mathbb{N}$, and $0 \leq v \leq 1$. Then

$$
\left\|\left(\mathbf{I}-\mathfrak{Z}_{v}\right)^{k} f\right\|_{p(\cdot)} \leq \mathbb{B} \cdot 2^{-1} v\left\|\left(\mathbf{I}-\mathfrak{Z}_{v}\right)^{k-1} f^{\prime}\right\|_{p(\cdot)}, \quad f \in W_{p(\cdot)}^{1},
$$

and

$$
\left\|\left(\mathbf{I}-\mathfrak{Z}_{v}\right)^{k} f\right\|_{p(\cdot)} \leq \mathbb{B}^{k} \cdot 2^{-k} v^{k}\left\|f^{(k)}\right\|_{p(\cdot)}, \quad f \in W_{p(\cdot)}^{k}
$$

hold.

\section{Transference RESUlt}

Here we state a variant of the transference result obtained in [5, Theorem 14]. Let $p \in \mathcal{P}, f \in L^{p(\cdot)}, G \in L^{p^{\prime}(\cdot)}$, and $\|G\|_{p^{\prime}(\cdot)}=1$. Note that the dual of $L^{p(\cdot)}$ is $L^{p^{\prime}(\cdot)}$. For any $\varepsilon>0$ there exists an $h_{1} \leq 1$ such that

$$
\Phi_{h_{1}} f(x)>f(x)-\varepsilon
$$


(see [5, (18)]). Everywhere in this work, this $h_{1}$ will be fixed. We define

$$
F_{f}(u):= \begin{cases}\left\langle\Phi_{h_{1}} f(\cdot+u), G\right\rangle=\int_{\mathbf{T}} \Phi_{h_{1}} f(x+u)|G(x)| d x, & \text { for }-h_{1} \leq u \leq h_{1}, \\ F_{f}\left(h_{1}\right) & \text { for } h_{1}<u \leq \pi, \\ F_{f}\left(-h_{1}\right) & \text { for } \pi \leq u<-h_{1} .\end{cases}
$$

Lemma 4.1 ([5, Lemma 13]). If $p \in \mathcal{P}, f \in L^{p(\cdot)}$, then the function $F_{f}(u)$ defined in (4.1) is uniformly continuous on $\left[-h_{1}, h_{1}\right]=: I_{h_{1}}$.

Theorem 4.2. Let $p \in \mathcal{P}$ and $f, g \in L^{p(\cdot)}$. If

then

$$
\left\|F_{g}\right\|_{C\left[I_{h_{1}}\right]} \leq \mathbf{c}\left\|F_{f}\right\|_{C\left[I_{h_{1}}\right]},
$$

$$
\|g\|_{p(\cdot)} \leq 3 \mathbf{c} \mathbb{B H}\|f\|_{p(\cdot)} .
$$

We define Peetre's $K$-functional

$$
K_{r}(f, v, X)_{X}:=\inf _{g \in X^{r}}\left\{\|f-g\|_{X}+v^{r}\left\|g^{(r)}\right\|_{X}\right\}, \quad v>0,
$$

and $K_{r}(f, v, p(\cdot)):=K_{r}\left(f, v, L^{p(\cdot)}\right)_{L^{p(\cdot)}}$ for $r \in \mathbb{N}, p \in \mathcal{P}, v>0$, and $f \in L^{p(\cdot)}$.

Proposition 4.3 ([5, Corollary 20]). If $0<h \leq v \leq 1$ and $f \in C[\mathbf{T}]$, the class of functions continuous on $\mathbf{T}$, then

$$
\left\|\left(\mathbf{I}-\mathfrak{Z}_{h}\right) f\right\|_{C[\mathbf{T}]} \leq 72\left\|\left(\mathbf{I}-\mathfrak{Z}_{v}\right) f\right\|_{C[\mathbf{T}]} .
$$

Lemma 4.4. Let $0<h \leq v \leq 1, p \in \mathcal{P}$, and $f \in L^{p(\cdot)}$. Then

$$
\frac{1}{3}\left\|\left(\mathbf{I}-\mathfrak{Z}_{h}\right) f\right\|_{p(\cdot)} \leq 72 \mathbb{B H}\left\|\left(\mathbf{I}-\mathfrak{Z}_{v}\right) f\right\|_{p(\cdot)} .
$$

5. Approximation By JaCKSON Operators

Proposition 5.1 ([24]). For every $n \in \mathbb{N}, p \in \mathcal{P}, f \in W_{p(\cdot)}^{1}$, the inequality

holds.

$$
E_{n}(f)_{p(\cdot)} \leq\left\|f-D_{n} f\right\|_{p(\cdot)} \leq \frac{\mathbb{B}}{n^{1}}\left\|f^{(1)}\right\|_{p(\cdot)}
$$

Theorem 5.2. If $p \in \mathcal{P}, f \in L^{p(\cdot)}$, and $n \in \mathbb{N}$, then

$$
\left\|f-D_{n} f\right\|_{p(\cdot)} \leq \mathbb{B}[216 \mathbb{H}+2[1+108 \mathbb{B} \mathbb{H}+432 \mathbb{B} \mathbb{H} \ln 2]+216 \mathbb{E} \mathbb{H}] \Omega_{1}\left(f, \frac{1}{n}\right)_{p(\cdot)} \text {. }
$$

To find an equivalence between the modulus of smoothness $\Omega_{r}(f, \delta)_{p(\cdot)}$ and the $K$-functional $K_{r}(f, \delta, p(\cdot))_{p(\cdot)}$ we consider the operator (see [20, 24]) defined for $f \in L^{p(\cdot)}, p \in \mathcal{P}$, as

$$
\left(\Re_{v} f\right)(x):=\frac{2}{v} \int_{v / 2}^{v}\left(\frac{1}{h} \int_{0}^{h} f(x+t) d t\right) d h, \quad x \in \mathbf{T}, v>0
$$


Note that for $0<v \leq 1, p \in \mathcal{P}$ we know that

$$
\left\|\Re_{v} f\right\|_{p(\cdot)} \leq \mathbb{B}\|f\|_{p(\cdot)},
$$

hence $f-\mathfrak{R}_{v} f \in L^{p(\cdot)}$ for $f \in L^{p(\cdot)}$. We set $\mathfrak{R}_{v}^{r} f:=\left(\mathfrak{R}_{v} f\right)^{r}$.

Lemma 5.3. Let $0<v, p \in \mathcal{P}$ and $f \in L^{p(\cdot)}$. Then

$$
\left\|\left(\mathbf{I}-\mathfrak{R}_{v}\right) f\right\|_{p(\cdot)} \leq 216 \mathbb{H} \mathbb{B}\left\|\left(\mathbf{I}-\mathfrak{Z}_{v}\right) f\right\|_{p(\cdot)} .
$$

Proof. If $f \in L^{p(\cdot)}$, we can use the generalized Minkowski integral inequality and Lemma 4.4 to obtain

$$
\begin{aligned}
\left\|\left(\mathbf{I}-\mathfrak{R}_{v}\right) f\right\|_{p(\cdot)} & =\left\|\frac{2}{v} \int_{v / 2}^{v}\left(\frac{1}{h} \int_{0}^{h}(f(x+t)-f(x)) d t\right) d h\right\|_{p(\cdot)} \\
& =\left\|\frac{2}{v} \int_{v / 2}^{v}\left(\mathfrak{Z}_{h} f(x)-f(x)\right) d h\right\|_{p(\cdot)} \leq \frac{2}{v} \int_{v / 2}^{v}\left\|\mathfrak{Z}_{h} f-f\right\|_{p(\cdot)} d h \\
& \leq 216 \mathbb{H B}\left\|\mathfrak{Z}_{v} f-f\right\|_{p(\cdot)} \frac{2}{v} \int_{v / 2}^{v} d h=216 \mathbb{H} \mathbb{B}\left\|\left(\mathbf{I}-\mathfrak{Z}_{v}\right) f\right\|_{p(\cdot)} .
\end{aligned}
$$

Remark 5.4. Note that the function $R_{v} f$ is absolutely continuous ([24]) and differentiable a.e. on $\mathbf{T}$.

Lemma 5.5. Let $0<v \leq 1, p \in \mathcal{P}$, and $f \in W_{p(\cdot)}^{1}$. Then

$$
\frac{d}{d x} \mathfrak{R}_{v} f(x)=\mathfrak{R}_{v} \frac{d}{d x} f(x) \quad \text { and } \quad \frac{d}{d x} \mathfrak{Z}_{v} f(x)=\mathfrak{Z}_{v} \frac{d}{d x} f(x), \quad \text { a.e. on } \mathbf{T} \text {. }
$$

Proof. The proof is the same as the proof of Lemma 24 of [5].

Lemma 5.6. Let $0<v \leq 1, p \in \mathcal{P}$, and $f \in L^{p(\cdot)}$ be given. Then

$$
v\left\|\frac{d}{d x} \mathfrak{R}_{v} f(x)\right\|_{p(\cdot)} \leq 2[1+108 \mathbb{B H}+432 \mathbb{B H} \ln 2]\left\|\left(\mathbf{I}-\mathfrak{Z}_{v}\right) f\right\|_{p(\cdot)} .
$$

Proof. For $f \in L^{p(\cdot)}$ by [24, p. 426] we know that

$$
\begin{aligned}
\varkappa:=\frac{v}{2} \| \frac{d}{d x} \mathfrak{R}_{v} f(x) & \left\|_{p(\cdot)} \leq\right\| \mathfrak{Z}_{v} f(x)-f(x)-\frac{1}{2}\left(\mathfrak{Z}_{v / 2} f(x)-f(x)\right) \|_{p(\cdot)} \\
& +\left\|\int_{v / 2}^{v} \frac{1}{h}\left(\mathfrak{Z}_{h} f(x)-f(x)-\frac{v}{2 h}\left(\mathfrak{Z}_{h / 2} f(x)-f(x)\right)\right) d h\right\|_{p(\cdot)}
\end{aligned}
$$

and hence

$$
\begin{aligned}
\varkappa \leq & \left\|\left(\mathbf{I}-\mathfrak{Z}_{v}\right) f\right\|_{p(\cdot)}+\frac{1}{2}\left\|\left(\mathbf{I}-\mathfrak{Z}_{v / 2}\right) f\right\|_{p(\cdot)} \\
& +\left\|\int_{v / 2}^{v} \frac{1}{h}\left(\left|\mathfrak{Z}_{h} f(x)-f(x)\right|+\frac{v}{2 h}\left|\mathfrak{Z}_{h / 2} f(x)-f(x)\right|\right) d h\right\|_{p(\cdot)} .
\end{aligned}
$$


One can find by Lemma 4.4 and the generalized Minkowski integral inequality:

$$
\begin{aligned}
\varkappa \leq & \left\|\left(\mathbf{I}-\mathfrak{Z}_{v}\right) f\right\|_{p(\cdot)}+\frac{1}{2}\left\|\left(\mathbf{I}-\mathfrak{Z}_{v / 2}\right) f\right\|_{p(\cdot)} \\
& +\left\|\int_{v / 2}^{v} \frac{1}{h}\left|\mathfrak{Z}_{h} f(x)-f(x)\right| d h\right\|_{p(\cdot)}+\left\|\int_{v / 2}^{v} \frac{1}{h}\left|\mathfrak{Z}_{h / 2} f(x)-f(x)\right| d h\right\|_{p(\cdot)} \\
& \leq(1+108 \mathbb{B H})\left\|\left(\mathbf{I}-\mathfrak{Z}_{v}\right) f\right\|_{p(\cdot)}+\int_{v / 2}^{v} \frac{1}{h}\left[\left\|\mathfrak{Z}_{h}-f\right\|_{p(\cdot)}+\left\|\mathfrak{Z}_{h / 2}-f\right\|_{p(\cdot)}\right] d h \\
& \leq(1+108 \mathbb{B H})\left\|\left(\mathbf{I}-\mathfrak{Z}_{v}\right) f\right\|_{p(\cdot)}+144 \mathbb{B} \mathbb{H}\left\|\mathfrak{Z}_{v} f-f\right\|_{p(\cdot)} \int_{v / 2}^{v} \frac{1}{h} d h \\
& \leq(1+108 \mathbb{B H}+432 \mathbb{B H} \ln 2)\left\|\left(\mathbf{I}-\mathfrak{Z}_{v}\right) f\right\|_{p(\cdot)}
\end{aligned}
$$

Lemma 5.7. Let $0<v \leq 1, r-1 \in \mathbb{N}, p \in \mathcal{P}$, and $f \in L^{p(\cdot)}$ be given. Then

$$
\frac{d^{r}}{d x^{r}} \Re_{v}^{r} f(x)=\frac{d}{d x} \Re_{v} \frac{d^{r-1}}{d x^{r-1}} \Re_{v}^{r-1} f(x), \quad x \in \mathbf{T} .
$$

Proof. The proof is the same as the proof of Lemma 26 of [5].

Proof of Theorem 1.4. Let $r=1, p \in \mathcal{P}$, and $f \in L^{p(\cdot)}$. Since

$$
K_{1}(f, v, p(\cdot))_{p(\cdot)} \leq\left\|f-\mathfrak{R}_{v} f\right\|_{p(\cdot)}+v\left\|\frac{d}{d x} \Re_{v} f(x)\right\|_{p(\cdot)},
$$

from Lemma 5.3 and $(5.3)$ we find

$$
K_{1}(f, v, p(\cdot))_{p(\cdot)} \leq 2(1+108 \mathbb{B H}+432 \mathbb{B H} \ln 2)\left\|\left(\mathbf{I}-\mathfrak{Z}_{v}\right) f\right\|_{p(\cdot)} .
$$

From Lemma 3.1 for $g \in W_{p(\cdot)}^{1}$,

$$
\Omega_{1}(f, v)_{p(\cdot)} \leq \Omega_{1}(f-g, v)_{p(\cdot)}+\Omega_{1}(g, v)_{p(\cdot)} \leq(1+\mathbb{B})\|f-g\|_{p(\cdot)}+\frac{\mathbb{B}}{2} v\left\|g^{\prime}\right\|_{p(\cdot)},
$$

and taking infimum on $g \in W_{p(\cdot)}^{1}$ in the last inequality we get

$$
\Omega_{1}(f, v)_{p(\cdot)} \leq(1+\mathbb{B}) K_{1}(f, v, p(\cdot))_{p(\cdot)}
$$

and the equivalence of $\left\|\left(\mathbf{I}-\mathfrak{Z}_{v}\right) f\right\|_{p(\cdot)}$ with $K_{1}(f, v, p(\cdot))_{p(\cdot)}$ is established.

Now we will consider the case $r>1$. For $r=2,3, \ldots$ we consider the operator (see [2])

$$
\mathcal{A}_{v}^{r}:=\mathbf{I}-\left(\mathbf{I}-\mathfrak{R}_{v}^{r}\right)^{r}=\sum_{j=0}^{r-1}(-1)^{r-j+1}\left(\begin{array}{l}
r \\
j
\end{array}\right) \mathfrak{R}_{v}^{r(r-j)} .
$$

From the identity $\mathbf{I}-\mathfrak{R}_{v}^{r}=\left(\mathbf{I}-\mathfrak{R}_{v}\right) \sum_{j=0}^{r-1} \mathfrak{R}_{v}^{j}$ we find

$$
\begin{aligned}
\left\|\left(\mathbf{I}-\mathfrak{R}_{v}^{r}\right) g\right\|_{p(\cdot)} & \leq\left(\sum_{j=0}^{r-1} \mathbb{B}^{j}\right)\left\|\left(\mathbf{I}-\mathfrak{R}_{v}\right) g\right\|_{p(\cdot)} \\
& \leq\left(216 \mathbb{B} \mathbb{H} \sum_{j=0}^{r-1} \mathbb{B}^{j}\right)\left\|\left(\mathbf{I}-\mathfrak{Z}_{v}\right) g\right\|_{p(\cdot)}
\end{aligned}
$$


when $0<v \leq 1, p \in \mathcal{P}$, and $g \in L^{p(\cdot)}$. Since $\left\|f-\mathcal{A}_{v}^{r} f\right\|_{p(\cdot)}=\left\|\left(\mathbf{I}-\mathfrak{R}_{v}^{r}\right)^{r} f\right\|_{p(\cdot)}$, a recursive procedure gives

$$
\left\|f-\mathcal{A}_{v}^{r} f\right\|_{p, \omega}=\left\|\left(\mathbf{I}-\mathfrak{R}_{v}^{r}\right)^{r} f\right\|_{p(\cdot)} \leq \cdots \leq\left(216 \mathbb{B} \mathbb{H} \sum_{j=0}^{r-1} \mathbb{B}^{j}\right)^{r}\left\|\left(\mathbf{I}-\mathfrak{Z}_{v}\right)^{r} f\right\|_{p(\cdot)} .
$$

On the other hand, using Lemmas 5.7 and 5.3 recursively,

$$
\begin{aligned}
v^{r}\left\|\frac{d^{r}}{d x^{r}} \mathfrak{R}_{v}^{r} f\right\|_{p(\cdot)} & =v^{r-1} v\left\|\frac{d}{d x} \mathfrak{R}_{v} \frac{d^{r-1}}{d x^{r-1}} \mathfrak{R}_{v}^{r-1} f\right\|_{p(\cdot)} \\
& \leq 2(1+108 \mathbb{B H}+432 \mathbb{B} \mathbb{H} \ln 2) v^{r-1}\left\|\left(\mathbf{I}-\mathfrak{Z}_{v}\right) \frac{d^{r-1}}{d x^{r-1}} \mathfrak{R}_{v}^{r-1} f\right\|_{p(\cdot)} \\
& \leq \ldots \\
& \leq[2(1+108 \mathbb{B H}+432 \mathbb{B H} \ln 2)]^{r-1} v\left\|\frac{d}{d x} \mathfrak{R}_{v}\left(\mathbf{I}-\mathfrak{Z}_{v}\right)^{r-1} f\right\|_{p(\cdot)} \\
& \leq[2(1+108 \mathbb{B H}+432 \mathbb{B H} \ln 2)]^{r}\left\|\left(\mathbf{I}-\mathfrak{Z}_{v}\right)^{r} f\right\|_{p(\cdot)} \cdot
\end{aligned}
$$

Thus

$$
\begin{aligned}
& K_{r}(f, v, p(\cdot))_{p(\cdot)} \leq\left\|f-\mathcal{A}_{v}^{r} f\right\|_{p(\cdot)}+v^{r}\left\|\frac{d^{r}}{d x^{r}} \mathcal{A}_{v}^{r} f(x)\right\|_{p(\cdot)} \\
& \leq 2\left[\max \left\{216 \mathbb{B} \mathbb{H} \sum_{j=0}^{r-1} \mathbb{B}^{j}, 2(1+108 \mathbb{B} \mathbb{H}+432 \mathbb{B} \mathbb{H} \ln 2)\right\}\right]^{r}\left\|\left(\mathbf{I}-\mathfrak{Z}_{v}\right)^{r} f\right\|_{p(\cdot)} .
\end{aligned}
$$

For the reverse of the last inequality, when $g \in W_{p(\cdot)}^{r}$, from Lemma 3.1

$$
\begin{aligned}
\Omega_{r}(f, v)_{p(\cdot)} & \leq(1+\mathbb{B})^{r}\|f-g\|_{p(\cdot)}+\Omega_{r}(g, v)_{p(\cdot)} \\
& \leq(1+\mathbb{B})^{r}\|f-g\|_{p(\cdot)}+2^{-r} \mathbb{B}^{r} v^{r}\left\|g^{(r)}\right\|_{p(\cdot)}
\end{aligned}
$$

taking infimum on $g \in W_{p(\cdot)}^{r}$ in 5.4 we get

$$
\Omega_{r}(f, v)_{p(\cdot)} \leq(1+\mathbb{B})^{r} K_{r}(f, v, p(\cdot))_{p(\cdot)},
$$

and hence

$$
\Omega_{r}(f, v)_{p(\cdot)} \approx K_{r}(f, v, p(\cdot))_{p(\cdot)}
$$




\section{Proofs of the Results}

\subsection{Proof of the transference result.}

Proof of Theorem 4.2. Let $p \in \mathcal{P}$ and $f \in L^{p(\cdot)}$. In this case,

$$
\begin{aligned}
\left\|F_{g}\right\|_{C\left[I_{h_{1}}\right]} & \leq \mathbf{c}\left\|F_{f}\right\|_{C\left[I_{h_{1}}\right]} \\
& =\mathbf{c}\left\|\int_{\mathbf{T}} \Phi_{h_{1}} f(x+u)|G(x)| d x\right\|_{C\left[I_{h_{1}}\right]} \\
& =\mathbf{c} \max _{u \in I_{h_{1}}}\left|\int_{\mathbf{T}} \Phi_{h_{1}} f(x+u)\right| G(x)|d x| \\
& \leq \mathbf{c H} \max _{u \in I_{h_{1}}}\left\|\Phi_{h_{1}} f(\cdot+u)\right\|_{p(\cdot)}\|G\|_{p^{\prime}(\cdot)} \leq \mathbf{c} \mathbb{H} \mathbb{B}\|f\|_{p(\cdot)},
\end{aligned}
$$

by Theorem 2.1

On the other hand, for any $\varepsilon, \eta>0$ and appropriately chosen $G \in L^{p^{\prime}(\cdot)}$ with $\langle g, G\rangle=\int_{\mathbf{T}} g(x) G(x) d x \geq \frac{1}{3}\|g\|_{p(\cdot)}-\varepsilon,\|G\|_{p^{\prime}(\cdot)}=1$, one can find

$$
\begin{aligned}
\left\|F_{g}\right\|_{C\left[I_{h_{1}}\right]} & \geq\left|F_{g}(0)\right| \geq \int_{\mathbf{T}} \Phi_{h_{1}} g(x)|G(x)| d x \\
& >\int_{\mathbf{T}} g(x)|G(x)| d x-\eta \int_{\mathbf{T}}|G(x)| d x \geq \frac{1}{3}\|g\|_{p(\cdot)}-\varepsilon-\eta \mathbb{H}\|1\|_{p(\cdot)} .
\end{aligned}
$$

Since $\varepsilon, \eta>0$ are arbitrary we have

$$
\left\|F_{g}\right\|_{C\left[I_{h_{1}}\right]} \geq \frac{1}{3}\|g\|_{p(\cdot)}, \quad \varepsilon, \eta \rightarrow 0^{+},
$$

and hence

$$
\frac{1}{3}\|g\|_{p(\cdot)} \leq\left\|F_{g}\right\|_{C\left[I_{h_{1}}\right]} \leq \mathbf{c} \mathbb{H} \mathbb{B}\|f\|_{p(\cdot)} .
$$

This gives the required result.

Proof of Lemma 4.4. Let $0<h \leq v \leq 1, p \in \mathcal{P}$, and $f \in L^{p(\cdot)}$. In this case, by (4.2) and Lemma 4.1 .

$$
\begin{aligned}
\max _{u \in I_{h_{1}}} \mid F_{\left(\mathbf{I}-\mathfrak{Z}_{h}\right) f(u) \mid} & =\max _{u \in I_{h_{1}}}\left|\int_{\mathbf{T}} \Phi_{h_{1}}\left(\mathbf{I}-\mathfrak{Z}_{h}\right) f(x+u)\right| G(x)|d x| \\
& =\max _{u \in I_{h_{1}}}\left|\left(\mathbf{I}-\mathfrak{Z}_{h}\right) \int_{\mathbf{T}} \Phi_{h_{1}} f(x+u)\right| G(x)|d x| \\
& =\max _{u \in I_{h_{1}}}\left|\left(\mathbf{I}-\mathfrak{Z}_{h}\right) F_{f}(u)\right| \stackrel{4.2}{\leq} 72 \max _{u \in I_{h_{1}}}\left|\left(\mathbf{I}-\mathfrak{Z}_{v}\right) F_{f}(u)\right| \\
& =72 \max _{u \in I_{h_{1}}}\left|\left(\mathbf{I}-\mathfrak{Z}_{v}\right) \int_{\mathbf{T}} \Phi_{h_{1}} f(x+u)\right| G(x)|d x| \\
& \leq 72 \max _{u \in I_{h_{1}}}\left|\int_{\mathbf{T}} \Phi_{h_{1}}\left(\mathbf{I}-\mathfrak{Z}_{v}\right) f(x+u)\right| G(x)|d x| \\
& =72 \max _{u \in I_{h_{1}}}\left|F_{\left(\mathbf{I}-\mathfrak{Z}_{v}\right) f}(u)\right|
\end{aligned}
$$




$$
\begin{aligned}
& \leq 72 \mathbb{H} \max _{u \in I_{h_{1}}}\left\|\Phi_{h_{1}}\left(\mathbf{I}-\mathfrak{Z}_{v}\right) f(x+u)\right\|_{p(\cdot)}\|G\|_{p^{\prime}(\cdot)} \\
& \leq 72 \mathbb{H} \mathbb{B}\left\|\left(\mathbf{I}-\mathfrak{Z}_{v}\right) f\right\|_{p(\cdot)} .
\end{aligned}
$$

On the other hand, for any $\varepsilon, \eta>0$ and appropriately chosen $G \in L^{p^{\prime}(\cdot)}$ with $\langle f, G\rangle \geq \frac{1}{3}\|f\|_{p(\cdot)}-\varepsilon$ and $\|G\|_{p^{\prime}(\cdot)}=1$, one can get

$$
\begin{aligned}
\max _{u \in I_{h_{1}}}\left|\left(\mathbf{I}-\mathfrak{Z}_{h}\right) F_{f}(u)\right| & \geq\left|\left(\mathbf{I}-\mathfrak{Z}_{h}\right) F_{f}(0)\right| \\
& \geq\left(\mathbf{I}-\mathfrak{Z}_{h}\right) \int_{\mathbf{T}} \Phi_{h_{1}} f(x)|G(x)| d x \\
& =\int_{\mathbf{T}} \Phi_{h_{1}}\left(\mathbf{I}-\mathfrak{Z}_{h}\right) f(x)|G(x)| d x \\
& >\int_{\mathbf{T}}\left(\mathbf{I}-\mathfrak{Z}_{h}\right) f(x)|G(x)| d x-\eta \mathbb{H}\|1\|_{p(\cdot)} \\
& \geq \frac{1}{3}\left\|\left(\mathbf{I}-\mathfrak{Z}_{h}\right) f\right\|_{p(\cdot)}-\varepsilon-\eta \mathbb{H}\|1\|_{p(\cdot)} .
\end{aligned}
$$

Since $\varepsilon, \eta>0$ are arbitrary we obtain

$$
\max _{u \in I_{h_{1}}}\left|\left(\mathbf{I}-\mathfrak{Z}_{h}\right) F_{f}(u)\right| \geq \frac{1}{3}\left\|\left(\mathbf{I}-\mathfrak{Z}_{h}\right) f\right\|_{p(\cdot)}
$$

and hence

$$
\frac{1}{3}\left\|\left(\mathbf{I}-\mathfrak{Z}_{h}\right) f\right\|_{p(\cdot)} \leq \max _{u \in I_{h_{1}}}\left|\left(\mathbf{I}-\mathfrak{Z}_{h}\right) F_{f}(u)\right| \leq 72 \mathbb{H} \mathbb{B}\left\|\left(\mathbf{I}-\mathfrak{Z}_{v}\right) f\right\|_{p(\cdot)} .
$$

\subsection{Proof of Jackson's inequalities.}

Proof of Theorem 1.2. Let $n, r \in \mathbb{N}$. For $g \in W_{p(\cdot)}^{r}$ we have by Theorem 1.3 that

$$
E_{n}(f)_{p(\cdot)} \leq E_{n}(f-g)_{p(\cdot)}+E_{n}(g)_{p(\cdot)} \leq\|f-g\|_{p(\cdot)}+\frac{\mathbb{B}}{n^{r}}\left\|g^{(r)}\right\|_{p(\cdot)} .
$$

Taking infimum in the last inequality with respect to $g \in W_{p(\cdot)}^{r}$, one can get, by (5.5),

$$
\begin{aligned}
E_{n}(f)_{p(\cdot)} & \leq \mathbb{B} K_{r}(f, 1 / n, p(\cdot))_{p(\cdot)} \\
& \leq 2 \mathbb{B}\left[\max \left\{216 \mathbb{B} \mathbb{H} \sum_{j=0}^{r-1} \mathbb{B}^{j}, 2[1+108 \mathbb{B} \mathbb{H}+432 \mathbb{B} \mathbb{H} \ln 2]\right\}\right]^{r} \Omega_{r}\left(f, \frac{1}{n}\right)_{p(\cdot)} \\
& =\mathbb{C} \Omega_{r}\left(f, \frac{1}{n}\right)_{p(\cdot)} .
\end{aligned}
$$

At this stage, we will use the method given by Natanson and Timan 21] to obtain 1.10. By Corollary 1.5 we have, for $\rho \leq n$,

$$
\Omega_{r}\left(f, \frac{1}{\rho}\right)_{p(\cdot)} \leq[1+\mathbb{B}]^{r} \frac{\mathbb{C}}{\mathbb{B}}\left[1+\frac{n}{\rho}\right]^{r} \Omega_{r}\left(f, \frac{1}{n}\right)_{p(\cdot)}
$$


and hence

$$
\begin{aligned}
\prod_{\rho=1}^{n} \Omega_{r}\left(f, \frac{1}{\rho}\right)_{p(\cdot)} & \leq \prod_{\rho=1}^{n}[1+\mathbb{B}]^{r} \frac{\mathbb{C}}{\mathbb{B}}\left[1+\frac{n}{\rho}\right]^{r} \Omega_{r}\left(f, \frac{1}{n}\right)_{p(\cdot)} \\
& =\left([1+\mathbb{B}]^{r} \frac{\mathbb{C}}{\mathbb{B}} \Omega_{r}\left(f, \frac{1}{n}\right)_{p(\cdot)}\right)^{n} \prod_{\rho=1}^{n}\left(1+\frac{n}{\rho}\right)^{r} .
\end{aligned}
$$

Using Stirling's formula we have

$$
\prod_{\rho=1}^{n}\left(1+\frac{n}{\rho}\right)^{r} \leq 2^{r} e^{r}
$$

and consequently

$$
\left(\prod_{\rho=1}^{n} \Omega_{r}\left(f, \frac{1}{\rho}\right)_{p(\cdot)}\right)^{1 / n} \leq(2 e[1+\mathbb{B}])^{r} \frac{\mathbb{C}}{\mathbb{B}} \Omega_{r}\left(f, \frac{1}{n}\right)_{p(\cdot)} .
$$

From Theorem 1.2 and the property $E_{n}(f)_{p(\cdot)} \downarrow$ as $n \uparrow$, we find

$$
\begin{aligned}
\left(\prod_{\rho=1}^{n} E_{\rho}(f)_{p(\cdot)}\right)^{1 / n} & \leq\left(\prod_{\rho=1}^{n} \mathbb{C} \Omega_{r}\left(f, \frac{1}{\rho}\right)_{p(\cdot)}\right)^{1 / n} \\
& \leq(2 e[1+\mathbb{B}])^{r} \mathbb{C}^{2} \mathbb{B}^{-1} \Omega_{r}\left(f, \frac{1}{n}\right)_{p(\cdot)}
\end{aligned}
$$

and the result 1.10 follows.

Proof of Theorem 1.3. Suppose that $\Theta_{n} \in \Pi_{n}, E_{n}\left(f^{\prime}\right)_{p(\cdot)}=\left\|f^{\prime}-\Theta_{n}\right\|_{p(\cdot)}$, and $\beta / 2$ is the constant term of $\Theta_{n}$, namely,

$$
\beta=\frac{1}{\pi} \int_{\mathbf{T}} \Theta_{n}(t) d t=\frac{1}{\pi} \int_{\mathbf{T}}\left(\Theta_{n}(t)-f^{\prime}(t)\right) d t
$$

We get

$$
|\beta / 2| \leq \frac{1}{2 \pi}\left\|f^{\prime}-\Theta_{n}\right\|_{1} \leq \frac{\mathbb{D}}{2 \pi}\left\|f^{\prime}-\Theta_{n}\right\|_{p(\cdot)}=\frac{\mathbb{D}}{2 \pi} E_{n}\left(f^{\prime}\right)_{p(\cdot)} .
$$

Furthermore,

$$
\begin{aligned}
\left\|f^{\prime}-\left(\Theta_{n}-\beta / 2\right)\right\|_{p(\cdot)} & \leq E_{n}\left(f^{\prime}\right)_{p(\cdot)}+\|\beta / 2\|_{p(\cdot)} \\
& \leq E_{n}\left(f^{\prime}\right)_{p(\cdot)}+\mathbb{D}(2 \pi)^{\frac{1}{\mathrm{p}}-1} E_{n}\left(f^{\prime}\right)_{p(\cdot)} \\
& =\left(1+\mathbb{D}(2 \pi)^{\frac{1}{\mathrm{p}}-1}\right) E_{n}\left(f^{\prime}\right)_{p(\cdot)} .
\end{aligned}
$$

We set $u_{n} \in \Pi_{n}$ so that $u_{n}^{\prime}=\Theta_{n}-\beta / 2$. Then

$$
\begin{aligned}
E_{n}(f)_{p(\cdot)} & =E_{n}\left(f-u_{n}\right)_{p(\cdot)} \leq \frac{\mathbb{B}}{n}\left\|f^{\prime}-\left(\Theta_{n}-\beta / 2\right)\right\|_{p(\cdot)} \\
& \leq \mathbb{B}\left(1+\mathbb{D}(2 \pi)^{\frac{1}{\mathrm{p}}-1}\right) \frac{1}{n} E_{n}\left(f^{\prime}\right)_{p(\cdot)} .
\end{aligned}
$$


The last inequality gives

$$
E_{n}(f)_{p(\cdot)} \leq\left[\mathbb{B}\left(1+\mathbb{D}(2 \pi)^{\frac{1}{\mathrm{p}}-1}\right)\right]^{r} \frac{1}{n^{r}} E_{n}\left(f^{(r)}\right)_{p(\cdot)} .
$$

Using Theorem 1.2 we obtain

$$
n^{r} E_{n}(f)_{p(\cdot)} \leq \mathbb{C}\left[\mathbb{B}\left(1+\mathbb{D}(2 \pi)^{\frac{1}{\mathrm{p}}-1}\right)\right]^{r} \Omega_{r}\left(f^{r}, \frac{1}{n}\right)_{p(\cdot)} .
$$

Proof of Theorem 5.2. From Proposition 5.1, Lemma 5.3 and Theorem 2.2 we get

$$
\begin{aligned}
\left\|f-D_{n} f\right\|_{p(\cdot)} & =\left\|f-\Re_{1 / n} f+\Re_{1 / n} f-D_{n} \Re_{1 / n} f+D_{n} \Re_{1 / n} f-D_{n} f\right\|_{p(\cdot)} \\
& \leq\left\|f-\Re_{1 / n} f\right\|_{p(\cdot)}+\left\|\Re_{1 / n} f-D_{n} \Re_{1 / n} f\right\|_{p(\cdot)}+\left\|D_{n}\left(\Re_{1 / n} f-f\right)\right\|_{p(\cdot)} \\
& \leq 216 \mathbb{B} \mathbb{H} \Omega_{1}\left(f, \frac{1}{n}\right)_{p(\cdot)}+\frac{\mathbb{B}}{n}\left\|\left(\Re_{1 / n} f\right)^{\prime}\right\|_{p(\cdot)}+\mathbb{E}\left\|\Re_{1 / n} f-f\right\|_{p(\cdot)} \\
& \leq \mathbb{B}[216 \mathbb{H}+2(1+108 \mathbb{B} \mathbb{H}+432 \mathbb{B} \mathbb{H} \ln 2)+216 \mathbb{E} \mathbb{H}] \Omega_{1}\left(f, \frac{1}{n}\right)_{p(\cdot)}
\end{aligned}
$$

and 5.1 follows.

\section{ACKNowledGements}

The author is indebted to the referees for their valuable suggestions and comments.

\section{REFERENCES}

[1] R. Akgün, Polynomial approximation in weighted Lebesgue spaces, East J. Approx. 17 (2011), no. 3, 253-266. MR 2953079

[2] R. Akgün, Trigonometric approximation of functions in generalized Lebesgue spaces with variable exponent, Ukrainian Math. J. 63 (2011), no. 1, 1-26. MR 2958823

[3] R. Akgün, Polynomial approximation of functions in weighted Lebesgue and Smirnov spaces with nonstandard growth, Georgian Math. J. 18 (2011), no. 2, 203-235. MR 2805977.

[4] R. Akgün, Approximation by polynomials in rearrangement invariant quasi Banach function spaces, Banach J. Math. Anal. 6 (2012), no. 2, 113-131. MR 2945992

[5] R. Akgün, Nikol'skiı̌, Jackson and Ul'yanov type inequalities with Muckenhoupt weights, arXiv:1709.02928v2 [math.CA].

[6] R. Akgün and V. Kokilashvili, On converse theorems of trigonometric approximation in weighted variable exponent Lebesgue spaces, Banach J. Math. Anal. 5 (2011), no. 1, 70-82. MR 2738521

[7] R. A. Bandaliev, Application of multidimensional Hardy operator and its connection with a certain nonlinear differential equation in weighted variable Lebesgue spaces, Ann. Funct. Anal. 4 (2013), no. 2, 118-130. MR 3034935

[8] S. O. Chaichenko, Best approximations of periodic functions in generalized Lebesgue spaces, Ukrainian Math. J. 64 (2013), no. 9, 1421-1439. MR 3104875

[9] D. V. Cruz-Uribe, A. Fiorenza, Variable Lebesgue Spaces: Foundations and Harmonic Analysis, Birkhäuser/Springer, 2013. MR 3026953

[10] L. Diening, P. Harjulehto, P. Hästö and M. Růžička, Lebesgue and Sobolev Spaces with Variable Exponents, Lecture Notes in Mathematics 2017, Springer, 2011. MR 2790542

[11] Z. Ditzian and K. G. Ivanov, Strong converse inequalities, J. Anal. Math. 61 (1993), 61-111. MR 1253439 
[12] V. K. Dzyadyk and I. A. Shevchuk, Theory of Uniform Approximation of Functions by Polynomials, De Gruyter, 2008. MR 2447076

[13] A. Guven and D. M. Israfilov, Trigonometric approximation in generalized Lebesgue spaces $L^{p(x)}$, J. Math. Inequal. 4 (2010), no. 2, 285-299. MR 2761228

[14] D. M. Israfilov and A. Testici, Approximation problems in the Lebesgue spaces with variable exponent, J. Math. Anal. Appl. 459 (2018), no. 1, 112-123. MR 3730430.

[15] D. M. Israfilov and E. Yirtici, Convolutions and best approximations in variable exponent Lebesgue spaces, Math. Rep. (Bucur.) 18(68) (2016), no. 4, 497-508. MR 3592374.

[16] S. Z. Jafarov, S. M. Nikolskii type inequality and estimation between the best approximations of a function in norms of different spaces. Math. Balkanica (N.S.) 21 (2007), no. 1-2, 173-182. MR 2350727

[17] S. Z. Jafarov, Ul'yanov type inequalities for moduli of smoothness, Appl. Math. E-Notes 12 (2012), 221-227. MR 3003875

[18] H. Koç, Simultaneous approximation by polynomials in Orlicz spaces generated by quasiconvex Young functions, Kuwait J. Sci. 43 (2016), no. 4, 18-31. MR 3756955

[19] Y. Kolomoitsev, On moduli of smoothness and averaged differences of fractional order, Fract. Calc. Appl. Anal. 20 (2017), no. 4, 988-1009. MR 3684879

[20] N. X. Ky, Moduli of mean smoothness and approximation with $A_{p}$-weights, Ann. Univ. Sci. Budapest. Eötvös Sect. Math. 40 (1997), 37-48. MR 1632805

[21] G. I. Natanson and M. F. Timan, The geometric means of the sequence of best approximations. (Russian) Vestnik Leningrad. Univ. Mat. Mekh. Astronom. 1979, vyp. 4, 50-52, 123. MR 0564575

[22] I. I. Sharapudinov, On the uniform boundedness in $L^{p}(p=p(x))$ of some families of convolution operators, Math. Notes 59 (1996), no. 1-2, 205-212. MR 1391844

[23] I. I. Sharapudinov, Some problems in approximation theory in the spaces $L^{p(x)}(E)$. (Russian) Anal. Math. 33 (2007), no. 2, 135-153. MR 2333023

[24] I. I. Sharapudinov, Approximation of functions in $L_{2 \pi}^{p(\cdot)}$ by trigonometric polynomials, Izv. Math. 77 (2013), no. 2, 407-434. MR 3097572

[25] I. I. Sharapudinov, On direct and inverse theorems of approximation theory in variable Lebesgue and Sobolev spaces, Azerb. J. Math. 4 (2014), no. 1, 55-72. MR 3167901.

[26] T. N. Shakh-Emirov, On uniform boundedness of some families of integral convolution operators in weighted variable exponent Lebesgue spaces, Izv. Sarat. Univ. (N.S.) Ser. Mat. Mekh. Inform. 14 (2014), no. 4(1), 422-427.

[27] R. M. Trigub, Linear summation methods and absolute convergence of Fourier series. (Russian) Izv. Akad. Nauk SSSR Ser. Mat. 32 (1968), 24-49. MR 0223818

[28] S. S. Volosivets, Approximation of functions and their conjugates in variable Lebesgue spaces, Sb. Math. 208 (2017), no. 1-2, 44-59. MR 3598764

[29] Y. E. Yildirir and D. M. Israfilov, Approximation theorems in weighted Lorentz spaces, Carpathian J. Math. 26 (2010), no. 1, 108-119. MR 2676725

Ramazan Akgün ${ }^{凶}$

Balıkesir University, Faculty of Arts and Sciences, Department of Mathematics, Çă̆iş

Yerleşkesi, 10145, Balıkesir, Türkiye.

rakgun@balikesir.edu.tr

Received: December 7, 2017

Accepted: September 11, 2018 